\title{
COMPARATIVE STUDY OF DYNAMIC RESPONSE OF MONOLITHIC AND ROCKING COLUMNS UNDER EARTHQUAKE ACCELERATIONS USING FINITE ELEMENT ANALYSIS
}

\author{
Rudra Chauhan, Ratik Chavan, Sureil Gupte and Yuvraj Chavda \\ Civil Engineering Department/ Universal College of Engineering, Kaman Vasai (E), India
}

\begin{abstract}
Rocking column is one of the types of Seismic Isolation system (also known as Kinematic Base Isolator), which is a high grade, either free standing or centrally post tensioned, concrete column with curved faces of column which are discontinuous in nature. In the present work, a comparative study of dynamic response is carried out between Monolithic and Rocking columns using ABAQUS Software. The main objective of the work is to analyse a monolithic RCC column and free-standing rocking RCC column and compare the results depending upon the stress \& strain characteristics when subjected to horizontal acceleration. After analysis, it was found that rocking column maintains its structural integrity with possible face-edge crushing failures.
\end{abstract}

Keywords: ABAQUS Software, Base Isolation, Earthquake, Finite Element Analysis, Rocking Column

Cite this Article: Rudra Chauhan, Ratik Chavan, Sureil Gupte and Yuvraj Chavda, Comparative Study of Dynamic Response of Monolithic and Rocking Columns Under Earthquake Accelerations using Finite Element Analysis, International Journal of Advanced Research in Engineering and Technology, 10(2), 2019, pp. 422-430. $\mathrm{http} / / /$ iaeme.com/Home/issue/IJARET?Volume $=10 \&$ Issue $=2$

\section{INTRODUCTION}

Since the world has experienced numerous devastating earthquakes over the past few decades, resulting in increased loss of human life due to collapse of buildings and severe structural damages, the structures like residential buildings, lifeline, historical and industrial structures need to be designed very carefully to protect from earthquakes. Structural design approach using seismic response control is now widely accepted and is frequently applied in Civil Engineering. In many applications, elastic performance during large earthquakes is economically feasible and the methodology permits performance-based design criteria, now required in many modern seismic design codes. Seismic Isolation is a concept in which an isolation bearing is used to isolate the superstructure from ground movement. It is governed by a fundamental period shift to higher periods which brings a reduction in forces attracted by the 
Comparative Study of Dynamic Response of Monolithic and Rocking Columns Under Earthquake Accelerations using Finite Element Analysis

structure but at the cost of increased isolator displacements. However, relative displacement of superstructure, which is primarily responsible for damage in the superstructure, is decreased achieving intended response. Rocking column is one of the types of Seismic Isolation system (also known as Kinematic Base Isolator), which is a high grade, either free standing or centrally post tensioned, concrete column with curved faces of column which are discontinuous in nature.

In the present work, a comparative study of dynamic response is carried out between Monolithic and Rocking columns using ABAQUS Software. The main objective of the work is to analyse a monolithic RCC column and free-standing rocking RCC column under cyclic horizontal accelerations and then compare the results depending upon the stress \& strain characteristics.

ABAQUS is Finite Element Analysis (FEA) software used for modelling and analysis of mechanical and structural components. The problem of the situation in earthquake loading involves use of horizontal acceleration data against the time period. For such data, quasi-static $\&$ dynamic analysis is required. ABAQUS provides a good environment for such analysis in various applications of Civil Engineering like structural health monitoring.

\section{MODULES IN ABAQUS}

Various modules of the ABAQUS software are mentioned as below [1]

\subsection{Part}

Parts are the building blocks of an Abaqus/CAE model. Part module is used to create each part which is assembled later in the assembly module.

\subsection{Property}

Property module is use to define materials, beam section profiles, sections, composite layups, skin reinforcement, inertia on a part, springs and dashpots between two points or between a point and ground, material calibrations.

\subsection{Assembly}

Assembly module is used to create and modify the assembly. A model contains one main assembly, which is composed of instances of parts from the model.

\subsection{Step}

Step module is used to create analysis steps, specify output requests, specify adaptive meshing, and specify analysis control.

\subsection{Interaction}

Interaction module is used to define and manage the mechanical and thermal interactions between regions of a model, the interface region and coupling methods, analysis constraints between regions of a model, connector sections, and connector section assignments to model connectors, Inertia (point mass, rotary inertia, and heat capacitance) on regions of the model.

\subsection{Load}

Load module is used to define and manage loads, boundary conditions, predefined fields and load cases. 


\subsection{Mesh}

Mesh module contains tools that allow you to generate meshes on parts and assemblies created within Abaqus/CAE. In addition, the Mesh module contains functions that verify an existing mesh.

\subsection{Optimization}

Optimization module is used to optimize the topology or shape of your model given a set of objectives and a set of restrictions.

\subsection{Job}

Job module is use to create and manage analysis jobs and to view a basic plot of the analysis results. It is also used to create and manage adaptivity analyses and co-executions.

\subsection{Visualization}

Visualization module is used to view your model and the results of the analysis.

\subsection{Sketch}

Sketches are two-dimensional profiles that are used to help form the geometry defining an Abaqus/CAE native part.

\section{METHODOLOGY}

Modelling of monolithic RCC column and free standing RCC rocking column is carried out in the ABAQUS software with the help of the modules available in the software. The steps involved in modelling both the columns are mentioned below.

\subsection{Part}

\subsubsection{Monolithic Column}

For modelling of monolithic column, three different parts are created in part module. First is the column with support blocks at both ends (as single unit). Second is the rebar of required length and diameter, bent at both ends and third is the stirrup of required diameter. The parts created for monolithic column are shown in Fig. 1. Dimensions of the parts are given in Table 1.

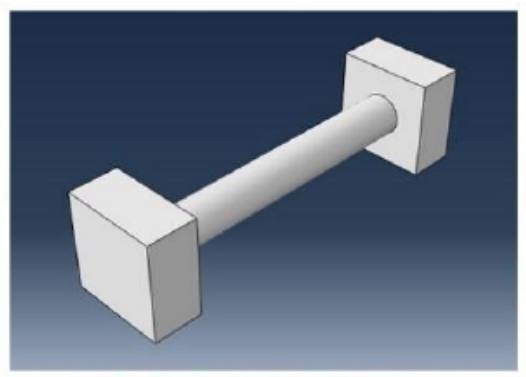

(a) Column with Support Blocks

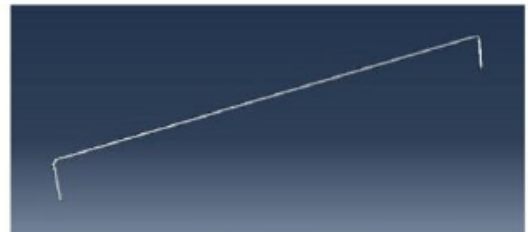

(b) Rebar

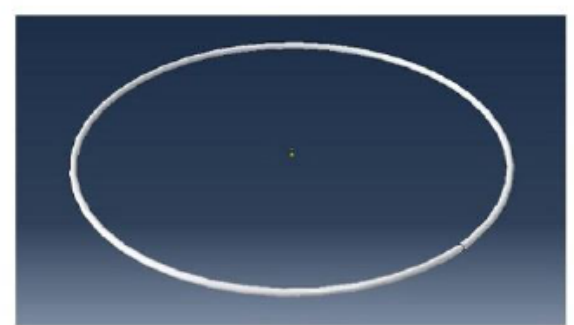

(c) Stirrup

Figure 1 parts of monolithic column 
Comparative Study of Dynamic Response of Monolithic and Rocking Columns Under Earthquake Accelerations using Finite Element Analysis

Table 1 Dimensions of elements of Monolithic Column

\begin{tabular}{|c|c|c|c|c|}
\hline & Support Blocks & Column & Stirrups & Rebar \\
\cline { 1 - 2 } Length & $1 \mathrm{~m}$ & \multirow{2}{*}{$0.425 \mathrm{~m}$ (Diameter) } & $\begin{array}{c}8 \mathrm{~mm} \phi \\
0.389 \mathrm{~m} \text { (Diameter) }\end{array}$ & $\begin{array}{c}16 \mathrm{~mm} \phi \\
7 \mathrm{nos} .\end{array}$ \\
\cline { 1 - 2 } Width & $1 \mathrm{~m}$ & $2.9 \mathrm{~m}$ & $250 \mathrm{~mm} \mathrm{c} / \mathrm{c} \mathrm{spacing}$ & $4.32 \mathrm{~m}$ \\
\hline
\end{tabular}

\subsubsection{Rocking Column}

For modelling of rocking column, four parts are created in part module i.e. column, support block, rebar and stirrup. For rocking column, column and support block are created separately. The parts created for rocking column are shown in Fig. 2. Dimensions of the Rocking Column are the same as that of Monolithic Column, except for the details of the column, rebars and stirrups. The bottom and the top face of the column is kept plane (flat) for the kern of the column, i.e. for the diameter of $106.25 \mathrm{~mm}$. A smooth curve is provided, tangential to the kern surface, intersecting the cylindrical surface at the height of $6.12 \mathrm{~mm}$ from the bottom as shown in Fig. 3; the dimensions are in millimetres. The height of the rebar is reduced to $2.82 \mathrm{~m}$ without the hook lengths.

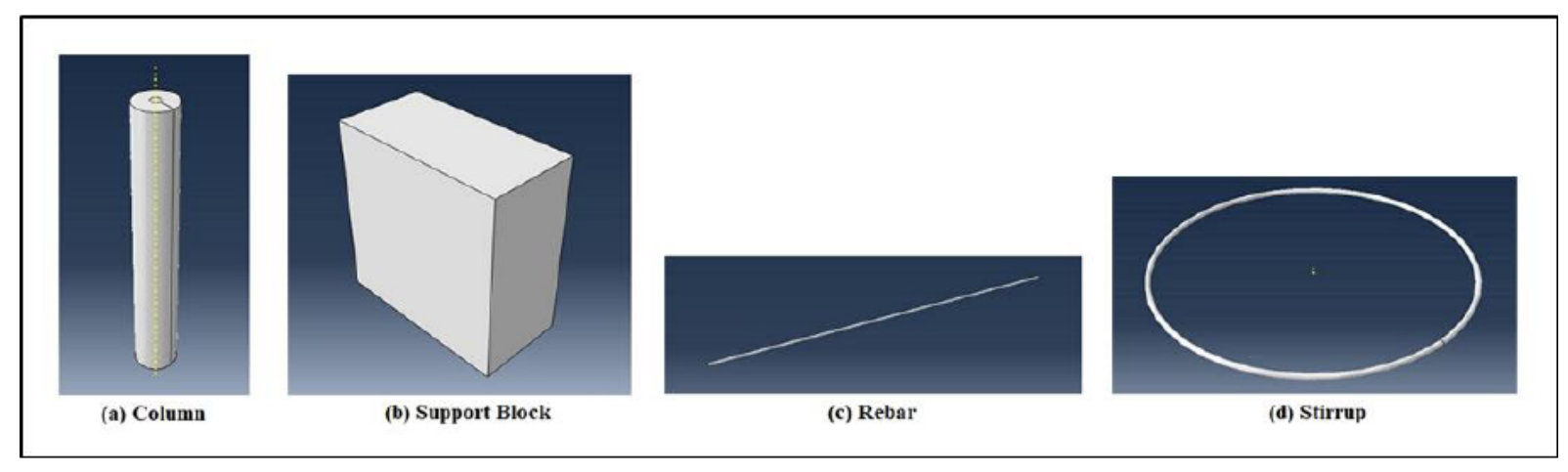

Figure 2 parts of rocking column

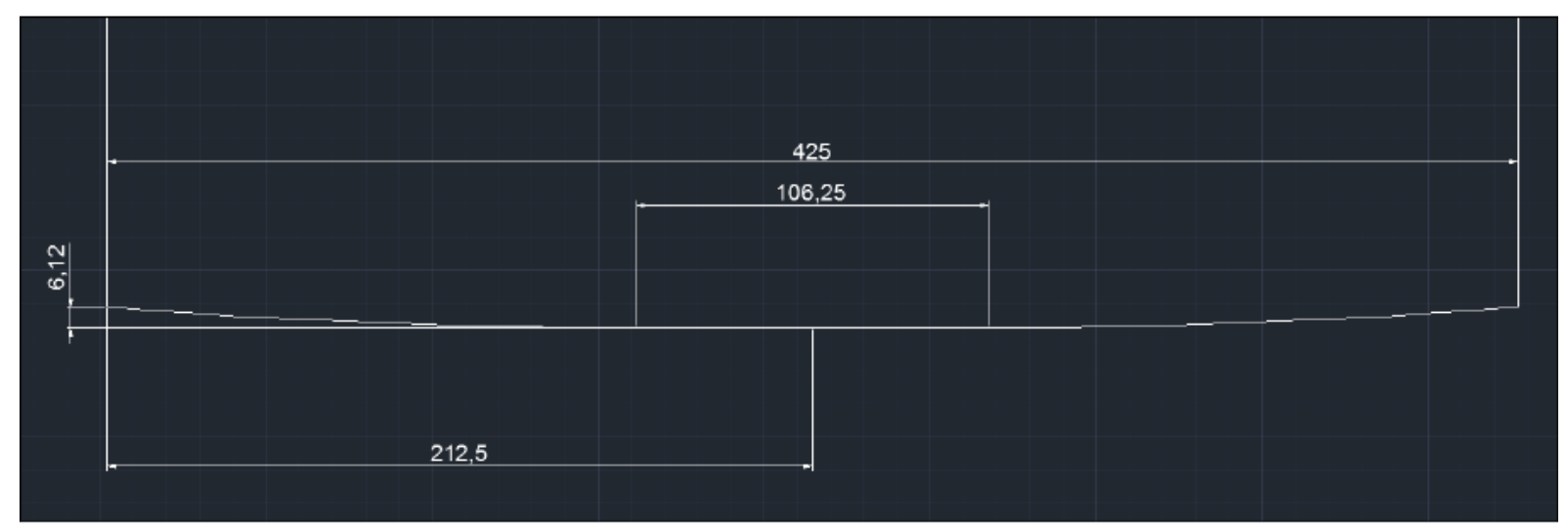

Figure 3 details of the curvature at the bottom $\&$ top face of the rocking column

\subsection{Property}

\subsubsection{Monolithic Column}

For monolithic column, two sections are created i.e. concrete and steel in the property module. For these sections a concrete material of M40 grade and a steel material of Fe500 are created and assigned to the sections and parts. 


\subsubsection{Rocking Column}

Similarly, for rocking column, two sections are created i.e. concrete and steel in the property module. For these sections a concrete material of M40 grade and a steel material of Fe500 are created and assigned to the sections and parts.

\subsection{Assembly}

\subsubsection{Monolithic Column}

In assembly module, first the instances of rebar and stirrup are created of required number. Then all the parts created in the part module are assembled together. During assembly, position of each part is fixed considering the spacing and dimension of the structure. Assembly of all the parts of the monolithic column is shown in Fig. 4.

\subsubsection{Rocking Column}

For rocking column, instances of support block, rebar and stirrup are created. Using the tools available, all the parts are assembled together and the position of each part is fixed. The rebars $\&$ stirrups are only in the column in case of rocking column and the block above and below are free.

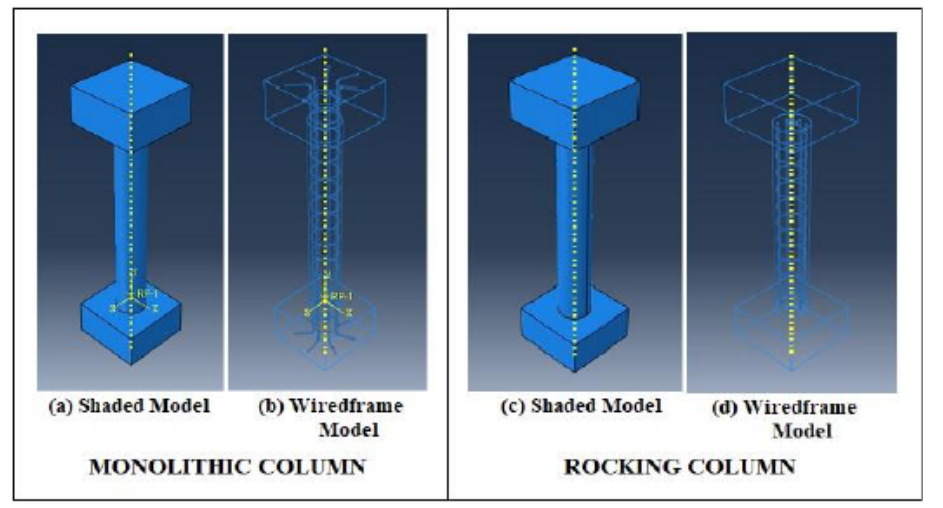

Figure 4 assembly of monolithic and rocking column

\subsection{Step}

In the step module, earthquake loading step is created. The field output request for Stress \& Strain with respect to time is specified for both monolithic and rocking column under Dynamic Analysis.

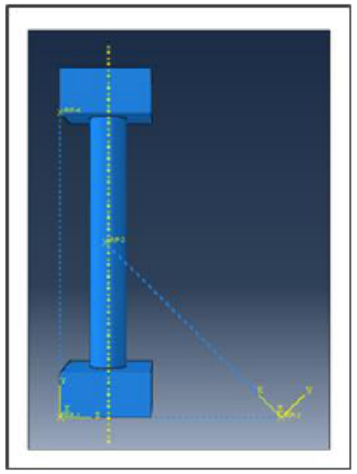

Figure 5 assembly of Rocking Column showing connectors

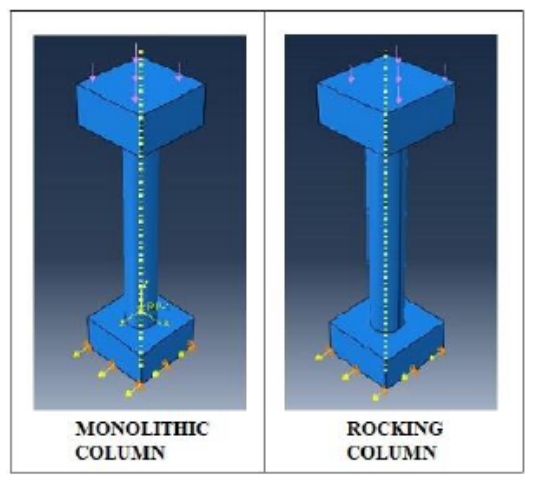

Figure 6 load and boundary condition for monolithic and rocking column 
Comparative Study of Dynamic Response of Monolithic and Rocking Columns Under Earthquake Accelerations using Finite Element Analysis

\subsection{Interaction}

\subsubsection{Monolithic Column}

In monolithic column, constraints are used with the help of interaction module. Firstly, the rebar and stirrups are embedded inside the column. Another constraint used is the rigid tie constraint which is applied using a reference point on the entire column.

\subsubsection{Rocking Column:}

In rocking column, interaction between the column and block is created at both the ends by selecting the bottom face of the column as master surface and top surface of bottom support block as slave surface; selecting the top face of the column as slave surface and the bottom surface of top support block as master surface. Now, Connectors are provided between the support blocks and the column such that the rotational parameters relative to the movement of the bottom block under earthquake accelerations are established as shown in Fig. 5.

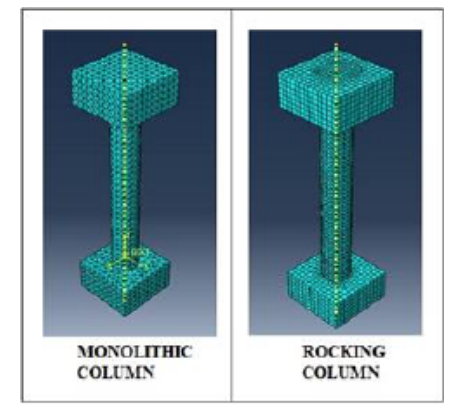

Figure 7 meshing of monolithic and rocking column

\subsection{Load}

At this stage a gravity load is given to the entire structure in both the cases. A pressure load of $1000 \mathrm{kN}$ is uniformly distributed at the top surface of the upper support block for both the columns. An earthquake load is placed at the bottom surface of the bottom support block. To create the earthquake conditions yet reduce the period of analysis, 2 consecutive cycles of horizontal accelerations against time are placed as earthquake loading. These accelerations are taken from the data acquired during the Vrancea earthquake which occurred on $4^{\text {th }}$ March, 1977 which was 7.2 Richter scale in magnitude. The load and boundary conditions for both the columns are shown in Fig. 6.

\subsection{Mesh}

All the parts created and assembled are meshed in the mesh module. The approximate mesh size for all the parts is $0.075 \mathrm{~m}$ which is the same in both monolithic and rocking column. But for the element shape, all the parts of the monolithic column have tet shape whereas in rocking column, support blocks have hex shape and rest all other parts are have tet shape. Meshing of both monolithic and rocking columns are shown in Fig. 7.

\section{ANALYSIS}

The above said procedure of modelling and analysis, which gave the required successful results was not determined easily and took more than 20 failed attempts and about 1700 hours of analysis time. A wait of about 60-70 hours was necessary to check the results and then accordingly determine the rectifications needed, implement the rectifications and run the analysis again. 
For the monolithic column, the graphical results were extracted for 3 particles placed at the bottom, middle and top respectively. Refer Fig. 4 for the orientations. If the column is viewed such that both $\mathrm{X} \& \mathrm{Y}$ axes are pointed towards positive directions, then the bottom particle lies at the junction of column and bottom support block facing towards the viewer. The middle particle lies exactly at the centre of the column within the mass of the column. And keeping the view orientation same, the top particle lies at the junction of column and top support block facing away from the viewer, i.e. on the other side of the column. For the rocking column, the graphical results were extracted for 3 particles placed exactly as described for the monolithic column.

These 3 particles were selected for both the monolithic and rocking columns in order to analyse evenly and compare the results by providing clear and invariable differences between the two columns.

\section{RESULTS}

Following are the results in graphical form; all left side graphs are for Monolithic Column referred as (a) and all right-side graphs for Rocking Column referred as (b)

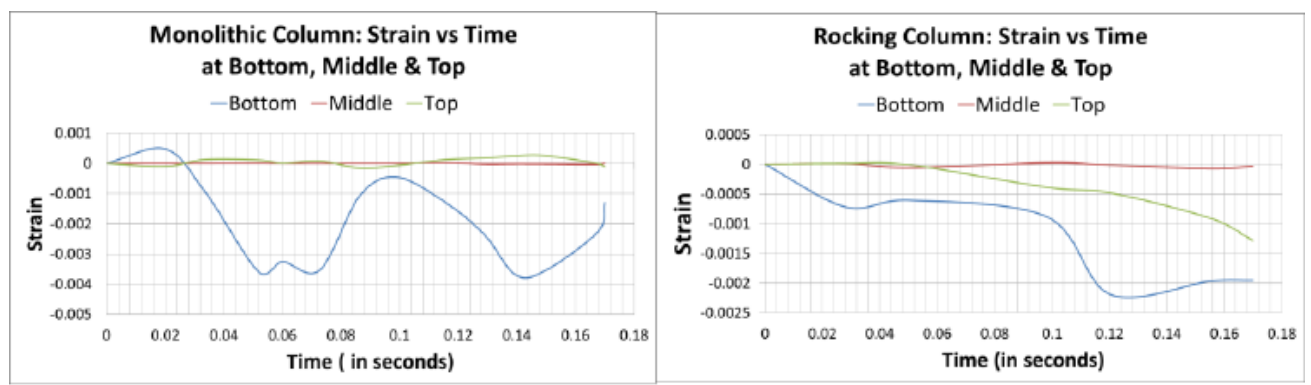

Figure 8 Strain vs Time curves at Bottom, Middle and Top for (a) and (b)

In Fig. 8, extreme variations in the strain-time curve are observed for the bottom particle for (a) whereas the same are observed for top and bottom particles for (b).

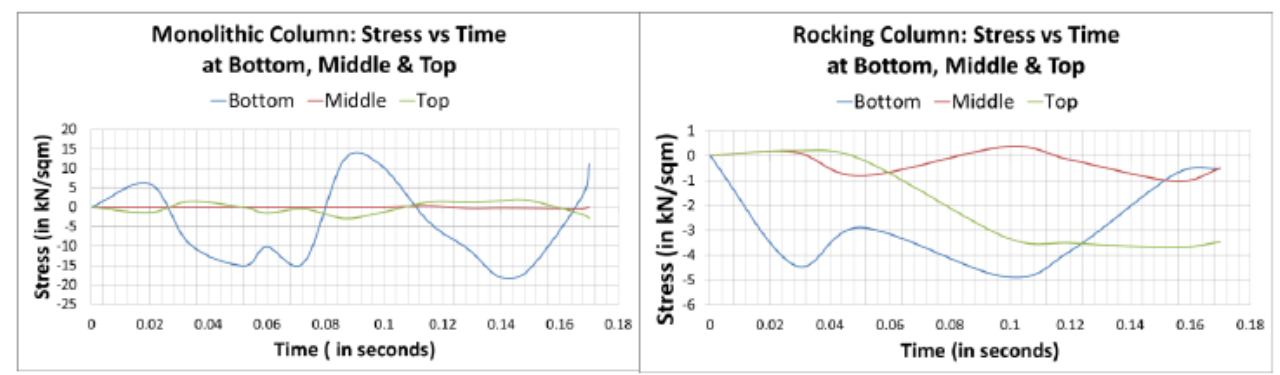

Figure 9 Stress vs Time curves at Bottom, Middle and Top for (a) and (b)

In Fig. 9, extreme variations in the stress-time curve are observed for the bottom particle for (a) whereas the same are observed for top and bottom particles for (b).

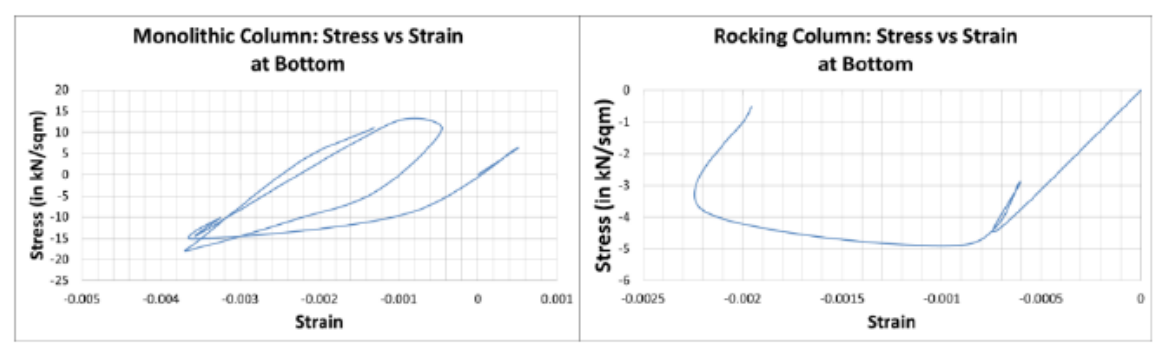

Figure 10 Stress vs Strain curve at Bottom particle for (a) and (b) 
Comparative Study of Dynamic Response of Monolithic and Rocking Columns Under Earthquake Accelerations using Finite Element Analysis

In Fig. 10, an unsteady multiple hysteresis loop type of stress-strain curve is observed for the bottom particle for (a) whereas a steady half hysteresis loop is observed for the bottom particle for (b).
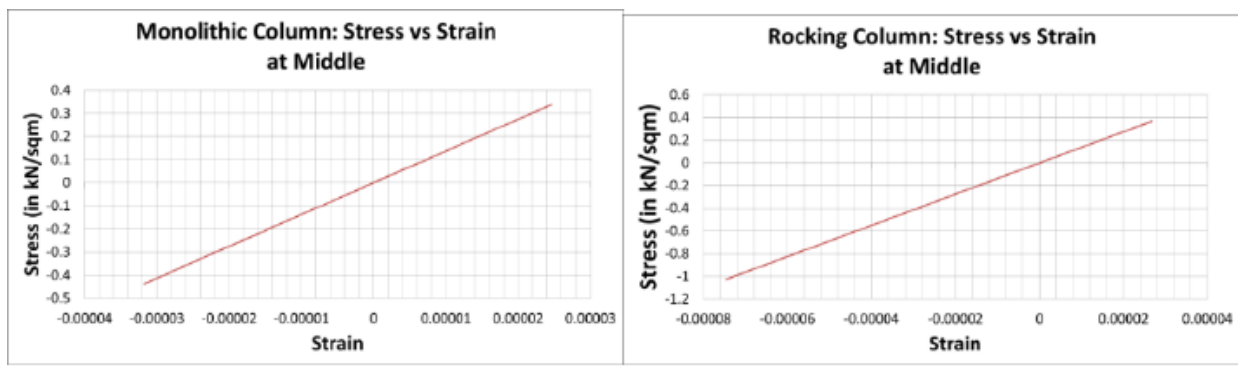

Figure 11 Stress vs Strain curve at Middle particle for (a) and (b)

In Fig. 11, although not visible at this scale, but if looked at closely, the stress-strain curves for middle particle for both (a) and (b) are extremely narrow and elongated hysteresis loops with very small stress $\&$ strain values.

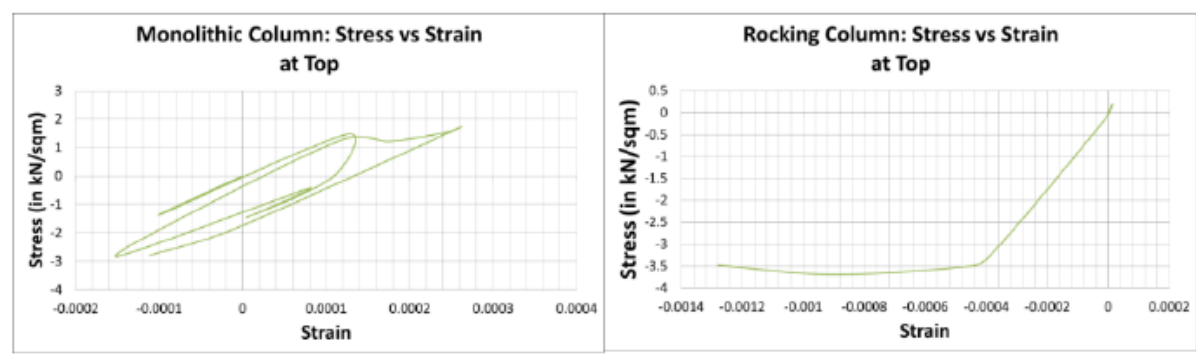

Figure 12 Stress vs Strain curve at Top particle for (a) and (b)

In Fig. 12, an unsteady multiple hysteresis loop type of stress-strain curve is observed for the top particle for (a) whereas a steady curve resembling to an inverted stress- strain curve for mild steel rod, is observed for the top particle for (b).

\section{CONCLUSION}

Following are the conclusions that can be drawn from the above obtained observations and results:

- From Fig. 8, it is clearly evident that the bottom portion for (a) experiences heavy variations in strain with respect to time, giving signs of deformation at the base of the column which indicates failure at the base for monolithic columns. For (b), it is observed to have steady increase in strain with respect to time at both top and bottom. This also indicates towards signs of failure at the edges of the rocking column.

- From Fig. 9, it is clearly evident that the bottom portion for (a) experiences heavy variations in stress with respect to time, some portions of the curve ranging in tensile stress region. This indicates the type of failure that occurs at the base; which can be established to be cracking and shearing. Also, (b) is observed to have steady increase in compressive stress with respect to time at both top and bottom. The steady increase in compression indicates failure due to crushing. Therefore, we get additional validation to the conclusions formed in previous point.

- From Fig. 10, 11 and 12, it is evident that monolithic column undergoes cyclic loading of narrow, elongated and multiple looped nature throughout the height of the column. Hence showing extreme, unsteady and rapid stress variations, with respect to time, induced in the column, indicative of higher probabilities of reduced durability, internal 
as well as surface cracks and structural failure. However, the rocking column undergoes cyclic loading of broad and half-looped nature throughout the height of the column. Hence showing steady and slow stress variations, with respect to time, induced in the column, indicative of sustained durability, structural integrity and lower probabilities of internal and surface cracks. However, due to extreme stresses developed at the face edges of the column indicate signs of crushing. This can be dealt with and overcame by provision of High Strength Steel Plates of prescribed curvature at the faces of the column and is included as the future scope of work along with analysis of rocking columns post-tensioned along the vertical centroid axis proven to improve its recentering capabilities.

\section{REFERENCES}

[1] Abaqus 6.13 Documentation, dsk.ippt.pan.pl/docs/abaqus/v6.13/index.html.

[2] Koh, Aik-Siong, and Ghulani Mustafa. "Free Rocking of Cylindrical Structures." Journal of Engineering Mechanics, vol. 116, no. 1, 1990, pp. 35-54., doi:10.1061/(asce)07339399(1990)116:1(35).

[3] Alessandro PALERMO, et al. "USE OF 'CONTROLLED ROCKING' IN THE SEISMIC DESIGN OF BRIDGES.” 13th World Conference on Earthquake Engineering, 2004.

[4] Desy Setyowulan, Keizo Yamamoto, ToshitakaYamao and Tomohisa Hamamoto, Dynamic Analysis of Concrete Girder Bridges Under Strong Earthquakes: The Effect of Collision, BaseIsolated Pier and Wing Wall, International Journal of Civil Engineering and Technology (IJCIET), Volume 6, Issue 4, April (2015), pp. 79-93

[5] R.S. Henry, et al. "Recentering Requirements for the Seismic Design of Self-Centering Systems." Proceedings of the Ninth Pacific Conference on Earthquake Engineering Building an Earthquake-Resilient Society 14-16 April, 2011, Auckland, New Zealand.

[6] Raid R. Al-Omari, Abdulaziz A. Al-Kifae and Sarmad M. AlTameemi, Earthquake Effect on Single Pile Behavior with Various Factor of Safety and Depth to Diameter Ratio in Liquefiable Sand, International Journal of Civil Engineering and Technology, 9(4), 2018, pp. 1253-1262

[7] Zhang, Qi, and M. Shahria Alam. "The Dynamics of Precast Post-Tensioned Rocking Columns." Structures Congress 2018, 2018, doi:10.1061/9780784481332.031.

[8] Sidramappa Shivashankar Dharane and Archita Vijaykumar Malge, Earthquake Resistant Ferrocement Hallow Columns and Cavity Walls - New Concept, International Journal of Civil Engineering and Technology (IJCIET), Volume 5, Issue 12, December (2014), pp. 292-295

[9] Makris, Nicos, and Michalis F. Vassiliou. "Sizing the Slenderness of Free-Standing Rocking Columns to Withstand Earthquake Shaking." Archive of Applied Mechanics, vol. 82, no. 10-11, 2012, pp. 1497-1511., doi:10.1007/s00419-012-0681-x.

[10] Dharane Sidramappa Shivashaankar and Patil Raobahdur Yashwant, Earthquake Resistant High-Rise Buildings -New Concept, International Journal of Advanced Research in Engineering and Technology (IJARET), Volume 5, Issue 6, June (2014), pp. 121-124

[11] Roh, Hwasung, and Andrei M. Reinhorn. "Nonlinear Static Analysis of Structures with Rocking Columns." Journal of Structural Engineering, vol. 136, no. 5, 2010, pp. 532-542., doi:10.1061/(asce)st.1943-541x.0000154.

[12] Sidramappa Shivashankar Dharane, Madhkar Ambadas Sul and Patil Raobahdur Yashwant, Earthquake Resistant RCC and Ferrocement Cicular Columns with Main Spiral Reinforcement, International Journal of Civil Engineering and Technology (IJCIET), Volume 5, Issue 9, September (2014), pp. 100-102

[13] Ming Narto Wijaya, Takuro Katayama, Ercan Serif Kaya and Toshitaka Yamao, Earthquake Response of Modified Folded Cantilever Shear Structure with Fixed-Movable-FixedsubFrames, International Journal of Civil Engineering and Technology (IJCIET), Volume 4, Issue 4, July-August (2013), pp. 194-207 